\title{
A NOVEL COMPUTATIONAL APPROACH OF IMAGE ANALYSIS TO QUANTIFY BEHAVIOURAL RESPONSE TO HEAT SHOCK IN CHIRONOMUS RAMOSUS LARVAE (DIPTERA: CHIRONOMIDAE)
}

\author{
BIMALENDU B. NATH ${ }^{1, *}$ and DAMAYANTI C. GHARPURE ${ }^{2}$ \\ ${ }^{1}$ Department of Zoology, Savitribai Phule Pune University, Pune-411007, India \\ 2 Department of Electronics Science, Savitribai Phule Pune University, Pune-411007, India \\ *Corresponding author: bbnath@gmail.com
}

\section{ABSTRACT}

\begin{abstract}
All living cells respond to temperature stress through coordinated cellular, biochemical and molecular events known as "heat shock response" and its genetic basis has been found to be evolutionarily conserved. Despite marked advances in stress research, this ubiquitous heat shock response has never been analysed quantitatively at the whole organismal level using behavioural correlates. We have investigated behavioural response to heat shock in a tropical midge Chironomus ramosus Chaudhuri, Das and Sublette. The filter-feeding aquatic Chironomus larvae exhibit characteristic undulatory movement. This innate pattern of movement was taken as a behavioural parameter in the present study. We have developed a novel computer-aided image analysis tool "Chiro" for the quantification of behavioural responses to heat shock. Behavioural responses were quantified by recording the number of undulations performed by each larva per unit time at a given ambient temperature. Quantitative analysis of undulation frequency was carried out and this innate behavioural pattern was found to be modulated as a function of ambient temperature. Midge larvae are known to be bioindicators of aquatic environments. Therefore, the "Chiro" technique can be tested using other potential biomonitoring organisms obtained from natural aquatic habitats using undulatory motion as a behavioural parameter.
\end{abstract}

Keywords: Chironomidae, Chironomus ramosus, insect behaviour, Chiro, undulation, heat shock, image analysis

\section{Introduction}

Living organisms have evolved mechanisms to adapt to environmental changes and survive under diverse forms of stress. Numerous studies over the last couple of decades have established that one of the responses to environmental stress, known as the "heat shock response", is the universal fundamental mechanism, necessary for organism's cellular homeostasis under unfavourable conditions (Nover 1991; Maresca and Lindquist 1991; Feder and Hoffmann 1999; Park et al. 2005; Åkerfelt et al. 2010). At the cellular and molecular level, the heat shock response is characterized by increased expression of a battery of genes, which leads to rapid accumulation of the heat shock proteins (HSPs) after a sudden increase in the ambient temperature. HSPs are expressed in response to a variety of stressors including temperature, heavy metals, oxygen radicals, ethanol, amino acid analogues and are highly conserved proteins among the prokaryotes and the eukaryotes (Schlesinger et al. 1990; Morimoto et al. 1994; Nover and Scharf 1997; Feder and Hoffmann 1999; Santoro 2000).

In natural habitats, temperature fluctuations are quite a common phenomenon which living populations are exposed to. With reference to ambient temperature, poikilotherms exhibit behavioural responses leading to homeostasis. Behavioural thermoregulatory responses and activity pattern have been reported in many organisms, especially in insects and crustaceans (Heath 1970; Slonim 1979; Heinrich 1981, 1996; Re and Dunham 1983; Wig- gins and Frappell 2002). An ecological approach to study heat shock response in natural populations has also been reported in a limited number of studies (Feder and Hoffmann 1999). While most investigators have focused on the cellular and molecular mechanisms underlying heat shock response, behavioural responses to heat stress at the whole organismal level has been underestimated. The vast literature on heat shock response accumulated over the last four decades, lack reports of any critical studies related to whole-organism's integrated response to heat stress. In order to address this issue, we have chosen an insect model to explore the behavioural responses to heat shock. In this paper, we have described the quantification of whole organismal response to heat shock in Chironomus larvae by an image analysis tool "Chiro", developed by us.

Chironomus, popularly known as midges, are unique insects of the order Diptera (Family: Chironomidae) that can thrive in all types of extreme aquatic habitats from polar, to temperate, to subtropical and tropical environments (Pinder 1986; Armitage et al. 1995). Chironomid midges show adaptations to a wide range of temperature fluctuations (Oliver 1971; Ferrington 2008) and appropriately qualify as models to study temperature stress. Previously, several works on HSPs and heat shock inducible chromosomal loci were reported mainly in two European species, namely, Chironomus tentans Fabricius and Chironomus thummi (Kieffer) (Tanguay and Vincent 1981; Lezzi 1984; Carretero et al. 1991; Sass 1995; Martinez et al. 1997; Morcillo et al. 1997) and in one Indian 
species, Chironomus striatipennis Kieffer (Nath and Lakhotia 1989). In the present study, a tropical Indian species, Chironomus ramosus Chaudhuri, Das and Sublette, colonized in the laboratory (Nath and Godbole 1998) has been chosen to study behavioural response to heat shock.

Aquatic Chironomus larvae are tubicolous. They build individual "housing tubes" and show characteristic undulating movements inside the tube. This undulating behaviour helps the larva to draw water current across the tube, which constitutes an efficient filter-feeding mechanism (Armitage et al. 1995). In the present study, larval "undulatory movement" was taken as a behavioural parameter and the frequency of undulation was measured as a function of ambient temperature using "Chiro", which is a computer-aided imaging tool developed by us for the quantification of behavioural responses to heat shock.

\section{Material and Methods}

\section{Experimental animals}

Midge larvae belonging to the species Chironomus ramosus were used. Larvae were originally obtained from an isofemale line of S/S wild type population following a breeding scheme described by Hardikar and Nath (2001). All developmental stages were maintained in the laboratory by using a mass rearing technique (Nath and Godbole 1998) and fourth instar larvae from the synchronous culture were used for the present study.

\section{Undulatory behaviour}

A fourth instar larva was picked up from the culture and put into a small glass Petri dish with water and sand particles. The larva completed making a housing tube within an hour. Three small windows were made on the anterior, middle and posterior part of the tube using fine forceps so that the red-coloured larva could be clearly seen undulating its head, abdomen and posterior parapods inside the tube.

During undulation, the wave like motion of the larval body starts from the head, traversing in an antero-posterior direction and ends at the tip of the posterior parapods. One complete movement from head to the posterior parapods constitutes one undulation. However, the larva does not undulate continuously. Under natural condition, a larva undulates for some time and then stops. We define the former as an "undulatory period" and cessation of movement as a "rest period", which is then followed by next phase of undulation.

\section{Behavioural assay with gradual temperature shift}

The experimental dish containing the larva within the tube was placed on a hotplate with thermostat control. Temperature of the water in the dish was monitored by a thermometer. The hotplate was switched on and the undulatory movement with every degree centigrade rise in temperature was captured as an image sequence. This was accomplished with the help of a CCD camera and image grabber as a function of time. The experiment was repeated for ten times and the mean undulatory frequency was plotted against every degree centigrade rise in temperature.

\section{Behavioural assay with sudden temperature shift}

In this assay, the experimental dish containing the larva was kept at room temperature. Undulatory movement and intermittent "rest" periods were continuously monitored. Heat shock treatment was given during the "rest" period by quickly replacing the water in the Petri dish with pre-warmed $\left(39^{\circ} \mathrm{C}\right)$ water. The experiment was repeated three times and the result was found to be reproducible.

\section{Image Analysis tool "Chiro"}

We have developed a package to measure the undulation frequency of Chironomus larvae, using image-processing techniques and named it "Chiro". The estimation of undulation frequency was automated through image acquisition and subsequent image processing.

During image acquisition, images were captured by a CCD camera with a resolution of $256 \times 256$ pixels and 256 grey shades and stored as sequential files namely image $^{1}$, image $^{2}$, image $^{3}, \ldots$, image $^{n}$. The images were initially pre-processed for the removal of noise using simple $3 \times 3$ median filter. Further linear contrast stretching was performed for obtaining an image with good contrast. Template matching technique (Puranik and Gharpure 2000) has been used to measure the undulation frequency of Chironomus larvae as described below.

As mentioned earlier, different temporal phases of larval undulation can be seen through the holes made on the "housing tube" schematically shown in Fig. 1. If the imagel showed the beginning of the undulatory phase from the anterior most point of larval head (Fig. 1a), then the tip of head with its typical features would be selected as "template" stored as $Y(i, j)$. The normalized correlation function has been used for the template matching. The correlation coefficient is given as:

$$
r_{x y}=\sum_{i=1}^{N} \sum_{j=1}^{N} X(i, j) Y(i, j)
$$

where $N \times N=$ size of template,

$X(i, j)=$ grey value of $(i, j)$-th pixel of sub-image underlying the template,

$Y(i, j)=$ grey value of $(i, j)$-th pixel of the template,

and the normalized correlation coefficient is

$$
\rho_{x y}=\frac{r_{x y}}{\sqrt{r_{x x} r_{y y}}}
$$

where 


$$
\begin{aligned}
& r_{x x}=\sum_{i=1}^{N} \sum_{j=1}^{N} X(i, j) X(i, j) \\
& r_{y y}=\sum_{i=1}^{N} \sum_{j=1}^{N} Y(i, j) Y(i, j)
\end{aligned}
$$

The coefficient $\rho_{x y}$ increases and is nearly 1 at the match positions. The software calculates $\rho_{x y}$ for template positions in the sequence of images to obtain the best match. The successive image files were then scanned sequentially for obtaining the "template match", indicating that the larva returned to the same position as grabbed in image (Fig. 1c). The best match position and correlation coefficients were stored in an array. The image in the sequence with the highest match coefficient was obtained. The sequential number of the image frame corresponding to a perfect match with the "template" was used to calculate the time elapsed between the two images with similar head position. Therefore, the frequency of undulation (f) was estimated from the frame capture rate as given below:

Time period of undulation $(t)=$ Number of images between two images showing perfect match $\times$ Time interval between two digitized images.

The frequency of undulation (f) is calculated as a reciprocal of the time period of undulation in hertz $(\mathrm{Hz})$. It was observed that template selection plays a vital role in measuring the undulation frequency.

\section{Results and Discussion}

The Chironomus larva undulates inside the housing tube. This undulation is one of the innate behavioural traits, chosen in the present study as a parameter to quantify the effect of heat shock response on the larva. Temperature stress was studied using two different methods. In one method the larvae were subjected to a gradual increase in ambient temperature and in the other method
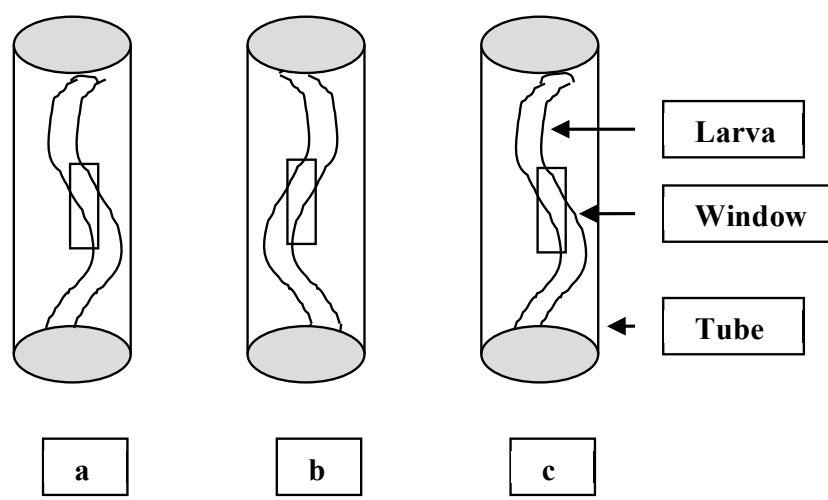

a

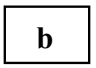

Fig. 1 Schematic showing temporal phases of larval undulation: (a) beginning (b) transient and (c) cessation of one undulatory movement. the larvae were given "heat shock" by exposing them to a sudden temperature shift from $25^{\circ} \mathrm{C}$ to $39^{\circ} \mathrm{C}$.

When larvae were exposed to a gradual temperature shift, $f$ values increased steadily showing a linear increase as a function of temperature. The $f$ value increased approximately by one fold beyond $30^{\circ} \mathrm{C}$ and larvae showed a two-fold rise in $f$ at $39^{\circ} \mathrm{C}$ (Fig. 2). Above $40^{\circ} \mathrm{C}$, the larvae were found to come out from the "housing tube" and showed aberrant swimming behaviour. These findings corroborated with the result reported in another tropical species of midge C. striatipennis, where $39^{\circ} \mathrm{C}$ was found to be the optimal threshold temperature for cytogenetic manifestation of heat shock response and above $39^{\circ} \mathrm{C}$, cell death was observed in laboratory reared larvae as evidenced by pycnotic nuclei (Nath and Lakhotia 1989). Our findings suggest that the threshold temperature for sensing the external temperature stress might be the same both at the cellular and at the whole organismal level.

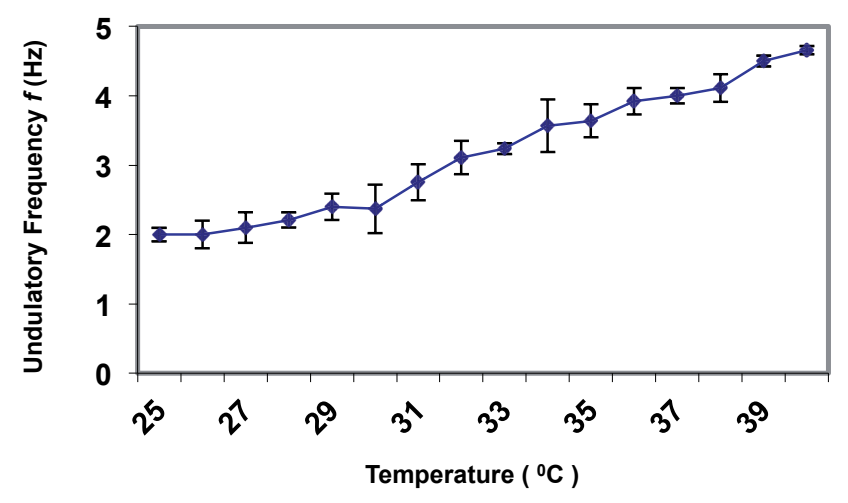

Fig. 2 Effect of gradual rise in ambient temperature on undulatory frequency.

In the second approach, a time-course experiment was conducted to study the behavioural effect of temperature stress when larvae were subjected to a sudden heat shock. The larval undulation ( $f$ values) continued to remain approximately constant at room temperature $\left(25^{\circ} \mathrm{C}\right)$ until the larva was subjected to sudden heat shock at $39^{\circ} \mathrm{C}$ at 90th second. Undulation frequency increased by one and half fold immediately after the heat shock treatment and increased further subsequently (Fig. 3). It is to be noted here that the time point of 90th second has been selected arbitrarily. The observed difference in the increment of $f$ values, between "gradual-shift" and "sudden-shift" data could be due to a cumulative effect of thermotolerance acquired by the larvae during the gradual rise in temperature. Interestingly, when we looked into the pattern of "rest intervals" followed by each phase of undulatory movement, an interesting profile was observed. Soon after the heat shock, the mean "rest interval" showed a four-fold decrease (Fig. 3). The larvae literally became "restless" and subsequently, came out of their tubes after 120th second. This assay also suggests that in real time analysis, $f$ values can be used as an indicator of a "sudden" rise in ambient temperature. 


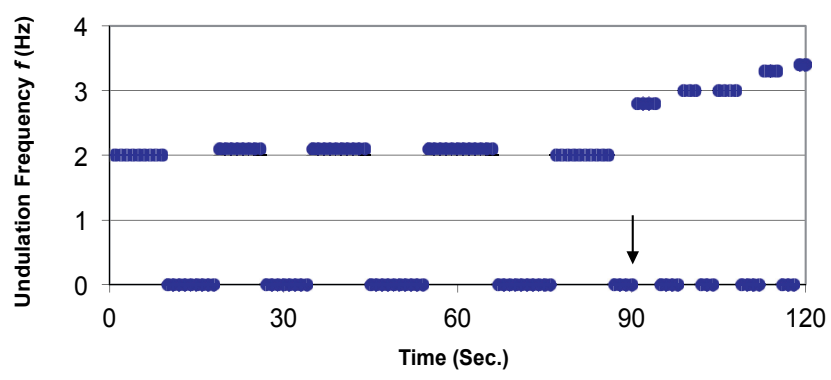

Fig. 3 Time course analysis of undulatory frequency of a larva before and after a shift in ambient temperature (heat shock) from $25{ }^{\circ} \mathrm{C}$ to $39^{\circ} \mathrm{C}$ at 90 th second.

Behavioural analysis and automatic data acquisition of body movements are becoming increasingly important in ethological and neurobiological research. However, the optoelectronic event recorders, which have been recently reported, are analogue based devices and utilize photo detectors (Hedwig 2000; Engel and Wyttenbach 2001). This has direct implications in elucidating an organism's response to ambient conditions. A few other attempts at designing specific software could be listed although general applicability of such software remains to be tested (Baek et al. 2002; Schwarz et al. 2002; Zakotnik et al. 2004; Cronin et al. 2005). In Chironomus, Brackenbury (2000) developed a video-recording based approach to monitor undulatory movement but did not explore its applicability in stress response studies. The past two decades have witnessed a rapid development of imaging technology, available to biologists, which enabled application of motion analysis in a wide range of organisms (Lopez-de-Victoria et al. 1995; Amsler et al. 2006) including the embryonic stages of zebrafish, a toad and a snail (Tills et al. 2013). However, all these methodologies reported so far are yet to be tested for wider applicability rather than for their taxon-specific customized usage.

As mentioned previously, little attention has been paid to behavioural studies for understanding "heat shock response" at the whole organismal level. However, a few studies related to other types of environmental stress are noteworthy. Depledge et al. (1995) described a computer-aided behavioural monitoring system to quantify changes in behavioural parameters due to metal toxicity. They utilised the "Shore crab" Carcinus maenas (Linnaeus) to study the effect of trace metal toxicity and selected parameters such as distance traveled, average speed, turning frequency, etc. Styrischare et al. (1995) reported disruption of circadian rhythms in the "Cray fish" Astacus astacus (Linnaeus) exposed to metal pollutants. A commonly available software (Image J) was used as an image analysis system by Untersteiner et al. (2005) which captured behaviour of multiple animals at a time. However, inferences could be obtained after a great deal of optimization and manual correction. Moreover, this video image analyser would be suitable for tracking trajectory of locomotor behaviour and not for analysis of single harmonic motion as displayed by a midge larva described in this paper. Magalhäes et al. (2007) reported a system of behavioural responses in zebrafish to chemical toxicity caused due to sodium hypochlorite using an image analysis system. The alteration of swimming activity could be detected by the biomonitoring system. However, image analysis protocol is altogether different and labour-intensive when compared to the one described by us in this paper.

In the present study, behavioural assays using Chironomus larvae has indicated that the undulatory movement can be considered as a reliable behavioural biomarker in midges to analyse heat shock response at the whole organismal level. Our study is the first attempt of its kind to analyse response to heat stress using behavioural parameters. Moreover, at the whole organismal level, no behavioural assays have been reported so far to quantify temperature induced motility changes in insects. In general, most organisms operate optimally very close to their temperature limit and at higher temperatures they exhibit increased motor activity. The pattern of such altered behaviour appears to be species-specific and identification of such pattern can be useful for "biomonitoring" environmental parameters. In other model organisms, analysis of similar behavioural indices should provide avenues for identifying new behavioural biomarkers for assessing environmental changes. More behavioural studies on stress response along with cellular and molecular analysis will eventually lead to an integrated approach to stress response research in future.

\section{Conclusions}

In this study, we have investigated behavioural response to heat shock in the larvae of a tropical midge, Chironomus ramosus, using its characteristic undulatory movement as a parameter for assessment. Quantification of frequency of undulation was monitored using a imaging technique "Chiro" developed by us. The experimental data revealed proportionate changes in the undulatory frequency along with the temperature of the surrounding aquatic medium and therefore, larval undulation frequency can be a reliable parameter for aquatic environmental biomonitoring which has been found to be modulated as a function of ambient temperature.

\section{Acknowledgements}

We thank Mr. Akash Mohabey for valuable inputs in developing image analysis software "Chiro" and Sucheta Deo for assisting in behavioural assays and data acquisition. BBN acknowledges partial financial support received from the Department of Zoology (UGCCAS Phase II grant, DST-PURSE grant and BCUD-UoP grant) and necessary supports from the Department of Electronic Sciences, University of Pune. 


\section{REFERENCES}

Åkerfelt M, Morimoto RI, Sistonen L (2010) Heat shock factors: integrators of cell stress, development and lifespan. Nat Rev Mol Cell Biol 11: 545-555.

Amsler MO, Amsler CD, Rittschof D, Becerro MA, McClintock JB (2006) The use of computer assisted motion analysis for quantitative studies of the behaviour of barnacle (Balanas amphitrite) larvae. Mar Freshw Behav Physiol 39: 259-268.

Armitage P, Cranston PS, Pinder LCV (eds) (1995) Chironomidae: Biology and Ecology of non-biting midges. Chapman and Hall, London.

Baek JH, Cosman P, Feng Z, Silver J, Schafer WR (2002) Using machine vision to analyze and classify Caenorhabditis elegans behavioural phenotypes quantitatively. J Neurosci Methods 118: 9-21.

Brackenbury J (2000) Locomotory modes in the larva and pupa of Chironomus plumosus (Diptera, Chironomidae). J Insect Physiol 46: 1517-1527.

Carretero MT, Carmona MJ, Diez JL (1991) Thermotolerance and heat shock proteins in Chironomus. J Insect Physiol 37: 239-246.

Cronin CJ, Mendel JE, Mukhtar S, Kim YM, Stirbl RC, Bruck J, Sternberg PW (2005) An automated system for measuring parameters of nematode sinusoidal movement. BMC Genet 6: 5 .

Depledge MH, Aagaard A, Györkös P (1995) Assessment of trace metal toxicity using molecular, physiological and behavioural biomarkers. Mar Pollut Bull 31: 19-27.

Engel JE, Wyttenbach RA (2001) An optoelectronic sensor for monitoring small movements in insects. Florida Ent 84: 336-343.

Feder M, Hoffmann GE (1999) Heat shock proteins, molecular chaperones and the stress response: evolutionary and ecological physiology. Ann Rev Physiol 61: 243-282.

Ferrington LC Jr (2008) Global diversity of non-biting midges (Chironomidae: Insecta-Diptera) in freshwater. Hydrobiol 595: 447-455.

Hardikar AA, Nath BB (2001) Chromosomal polymorphism is associated with nematode parasitism in a natural population of a tropical midge. Chromosoma 110: 58-64.

Heath JE (1970) Behavioural regulation of body temperature in poikilotherms. Physiologist 13: 399-410.

Hedwig B (2000) A highly sensitive optoelectronic system for the measurement of movements. J Neurosci Methods 100: 165-171.

Heinrich B (ed.) (1981) Insect thermoregulation. John Wiley and Sons, New York.

Heinrich B (1996) The thermal warriors: strategies of insect survival. Harvard University Press, Cambridge.

Lezzi M (1984) Heat shock phenomena in Chironomus tentans II. In vitro effect of heat and overheat on puffing and their reversal. Chromosoma 90: 198-203.

Lopez-de-Victoria G, Zimmerfaust RK, Lovell CR (1995) Computer assisted video motion analysis - a powerful technique for investigating motility and chemotaxis. J Microbial Methods 23: 329-341.

Magalhäes DP, Armando da Cunha R, Albuquerque dos Santos JA, Buss DF, Baptista DF (2007) Behavioural response of zebrafish Danio rerio Hamilon 1822 to sublethal stress by sodium hypochlorite: ecotoxicological assay using an image analysis biomonitoring system. Ecotoxicology 16: 417-422.

Maresca B, Lindquist S (eds) (1991) Heat shock. Springer Verlag, Berlin.

Martinez JL, Gorab E, Morcillo G, Diez, JL (1997) Screening of a micro-cloning library from heat shock induced TBR III of Chironomus thummi. Hereditas 127: 273.
Morcillo G, Gorab E, Tanguay RM, Diez, JL (1997) Specific intranucleolar distribution of HSP70 during heat shock in polytene cells. Exptl Cell Res 236: 361-370.

Morimoto RI, Tissieres A, Georgopolous C (eds) (1994) The biology of heat shock proteins and molecular Chaperones. Cold Spr Herb Press, New York.

Nath BB, Lakhotia SC (1989) Heat shock response and the effect of developmental stage and tissue type on heat shock protein synthesis. Genome 32: 461-475.

Nath BB, Godbole NN (1998) Technique for mass rearing of Indian Chironomus species (Diptera, Nematocera, Chironomidae). Studia Dipterologica 5: 187-193.

Nover L (ed.) (1991) Heat shock responses. CRC Press, Florida.

Nover L, Scharf KD (1997) Heat stress proteins and transcription factors. Cell Mol Life Sci 53: 80-103.

Oliver DR (1971) Life history of Chironomidae. Ann Rev Ent 16: 211-230.

Park HG, Han SI, Oh SY, Kang HS (2005) Cellular responses to mild heat stress. Cell Mol Life Sci 62: 10-23.

Pinder LCV (1986) Biology of freshwater Chironomidae. Ann Rev Ent 37: 1-23.

Puranik MS, Gharpure DC (2000) Use of neural network for automatic trajectory plotting. I E T E J Res 46: 375-379.

Re BS, Dunham DW (eds) (1983) Studies in adaptation : The behaviour of higher Crustacea. John Wiley and Sons, New York.

Santoro MG (2000) Heat shock factors and the control of the stress response. Biochem Pharmacol 59: 55-63.

Sass H (1995) Transcription of heat shock gene loci versus nonheat shock loci in Chironomus polytene chromosomes; evidence for heat-induced formation of novel putative ribonucleoprotein particles (hs RNPs) in the major heat shock puffs. Chromosoma 103: 528-538

Schlesinger MJ, Santoro MG, Garaci E (eds) (1990) Stress proteins: Induction and function. Springer Verlag, Berlin.

Schwarz S, Hoffmann MH, Gutzen C, Schlax S, von der Emde G (2002) VIEWER: a program for visualizing, recording, and analyzing animal behaviour. Comput Methods Programs Biomed 67: 55-66.

Slonim AD (1979) Homeostatic behaviour and behavioural homeostasis. Zh Obshch Biol 40: 118-127.

Styrishave B, Rasmussen AD, Depledge MH (1995) The influence of bulk and trace metals on the circadian rhythms of heart rates in freshwater cray fish Astacus astacus. Mar Pollut Bull 31: 87-92.

Tanguay RM, Vincent M (1981) Biosynthesis and characterisation of heat shock proteins in Chironomus tentans salivary glands. Can J Biochem Cell Biol 59: 67-73.

Tills O, Bitterli T, Culverhouse P, Spicer JI, Rundle S (2013) A novel application of motion analysis for detecting stress responses in embryos at different stages of development. BMC Bioinformatics 14: 37 .

Untersteiner H, Gretschel G, Puchner T, Napetschnig S, Kaiser H (2005) Monitoring behavioural responses to the heavy metal cadmium in the marine shrimp Hippolyte inermis Leach (Crustacea: Decapoda) with video imaging. Zool Studies 44: 71-80.

Wiggins PR, Frappell PB (2002) Behavioural thermoregulation in Daphnia carinata from different depths of a natural water body: influence of environmental oxygen levels and temperature. Comp Biochem Physiol A: Mol Integr Physiol 133: 771-780.

Zakotnik J, Matheson T, Durr V (2004) A posture optimization algorithm for model based motion capture of movement sequences. J Neurosci Methods 135: 43-54. 